\title{
A STURM-LIOUVILLE EIGENPROBLEM OF THE FOURTH KIND: A CRITICAL LATITUDE WITH EQUATORIAL TRAPPING *
}

\author{
JOHN P. BOYD \& ANDREI NATAROV ${ }^{\dagger}$
}

\begin{abstract}
Through both analytical and numerical methods, we solve the eigenproblem $u_{z z}+$ $\left(1 / z-\lambda-(z-1 / \epsilon)^{2}\right) u=0$ on the unbounded interval $z \in[-\infty, \infty]$ where $\lambda$ is the eigenvalue and $u(z) \rightarrow 0$ as $|z| \rightarrow \infty$. This models an equatorially-trapped Rossby wave in a shear flow in the ocean or atmosphere. It is the usual parabolic cylinder equation with Hermite functions as the eigenfunctions except for the addition of an extra term which is a simple pole. The pole, which is on the interior of the interval, is interpreted as the limit $\delta \rightarrow 0$ of $1 /(z-i \delta)$. The eigenfunction has a branch point of the form $z \log (z)$ at $z=0$ where the branch cut is on the upper imaginary axis. The eigenvalue is complex-valued with an imaginary part which we show, through matched asymptotics, is approximately $\sqrt{\pi} \exp \left(-1 / \epsilon^{2}\right)\{1-2 \epsilon \log \epsilon+\epsilon \log 2+\gamma \epsilon\}$. Because $\Im(\lambda)$ is transcendentally small in the small parameter $\epsilon$, it lies "beyond all orders" in the usual Rayleigh-Schrödinger power series in $\epsilon$. Nonetheless, we develop special numerical algorithms that are effective in computing $\Im(\lambda)$ for $\epsilon$ as small as $1 / 100$.
\end{abstract}

Key words. critical latitude, eigenproblem

AMS subject classifications.

1. Introduction. When the phase speed of a hydrodynamic wave matches the velocity of the shear flow through which it propagates, the linearized wave equation is singular. The surface where $U=c$ where $U$ is the mean current and $c$ is the wave's phase speed is called the "critical surface". Viscosity, nonlinearity or transience must smear out the singularity over an internal boundary layer of small but finite thickness.

Despite the long history of the subject as reviewed by Andrews, Holton and Leovy[2], there are still gaps in the theory. In this article, we examine the simplest problem for which (i) the singularity is resolved by weak dissipation and (ii) the wave is equatorially-trapped so that the amplitude of the wave at the critical surface is exponentially small in a parameter $\epsilon$ that measures the strength of the shear. This requires special analytical and numerical methods because the imaginary part of the eigenvalue is exponentially small in $1 / \epsilon$ and is thus invisible to a perturbation series in powers of $\epsilon$.

The simplest model for equatorially-trapped waves arises when the mean shear flow is a function only of latitude $y$. The linearized wave equations can be solved by the method of separation of variables. The latitudinal structure of the wave is given by a Sturm-Liouville eigenproblem.

Although our interest is in flows with a mean current $U(y)$, the special case of $U(y) \equiv 0$ is essential background. When there is no shear flow, and the geometry is approximated using the so-called "equatorial beta-plane", the eigenproblem is the parabolic cylinder equation on the infinite interval:

$$
u_{y y}+\left(-\lambda-y^{2}\right) u=0
$$

* This work was supported by the National Science Foundation through grant OCE9521133. This text is identical, except for typograpical corrections, with the article published in Studies in Applied Mathematics, vol. 101, no. 4, pgs. 433-455 (1998).

$\dagger$ Department of Atmospheric, Oceanic and Space Science and Laboratory for Scientific Computation, University of Michigan, 2455 Hayward Avenue, Ann Arbor MI 48109 (jpboyd@engin.umich.edu; http://www.engin.umich.edu:/ jpboyd/). 
The exact eigensolutions are

$$
\lambda=-(2 n+1), \quad u_{n}=\exp \left(-(1 / 2) y^{2}\right) H_{n}(y)
$$

where $n$ is an integer (the mode number) and $H_{n}(y)$ is a polynomial of degree $n$, the $n$-th Hermite polynomial. Because of the Gaussian factor $\exp \left(-(1 / 2) y^{2}\right)$, the eigenfunctions decay exponentially for large $|y|$ and are said to be "equatorially trapped".

In the presence of a mean east-west current $U(y)$, the wave equations are still linear and separable, but the eigenproblem is singular at the critical latitude where $U(y)=c$. In this work, we study the simplest example of a eigenproblem with both a critical latitude and equatorial trapping:

$$
u_{z z}+\left(1 / z-\lambda-(z-1 / \epsilon)^{2}\right) u=0 \quad \text { Hermite-with-Pole Equation }
$$

where

$$
z \equiv y-1 / \epsilon
$$

is a shifted latitudinal coordinate. For want of a standard name, we shall refer to this as the "Hermite-with-Pole" equation. Note that the actual equation for Rossby waves in a shear flow is more complicated than Eq.(3) $[5,6]$.

Singular eigenproblems have been studied by many authors including one of us $[5,6,7]$ as reviewed by Adams[1]. Boyd[7] dubbed such problems, where there was a singularity internal to the interval, as Sturm-Liouville Eigenproblems of the "Fourth Kind". However, equatorial trapping, which makes the imaginary part of the eigenvalue transcendentally small in the perturbation parameter $\epsilon$, introduces a whole slew of complications which have not been previously analyzed except for the limited work of Boyd and Christidis[12, 13]. They showed that the equatorial Kelvin wave has an instability in which the imaginary part of the phase speed (i. e., the growth rate), is an exponentially small function of the strength of the shear.

Our long-term goal is to return to the Kelvin instability to prove analytically what was demonstrated only through heuristic arguments and crude numerical solutions in the earlier work. Because of the formidable technical difficulties, we have chosen to begin with the much simpler problem of the Hermite-with-Pole equation.

For this, the imaginary part of the eigenvalue describes the rate at which the amplitude of the wave decays with height due to absorption of wave energy at the singularity. It is thus important even when very small. Because the imaginary part of the eigenvalue is transcendentally small compared to any finite power of $\epsilon$, we must employ the special methods of "beyond all orders" perturbation theory[16] and/or equally special numerical algorithms.

The analytical approximation has three key ingredients. The first is the "optical theorem", to borrow the term applied to similar relationships in particle physics, which shows that the imaginary part of the eigenvalue can be obtained by merely evaluating the eigenfunction at its singularity (plus a couple of easy additional steps) (Sec. 2). The second ingredient is a trio of local approximations: a Rayleigh-Schrödinger perturbation series in powers of $\epsilon$ which is accurate near the equator, a second in the region between the critical latitude and the equator, and one near the pole of the differential equation in terms of Whittaker functions (Secs. 3, 4 and 5). The third ingredient is asymptotic matching, which links the three local approximations in regions of mutual overlap.

The first numerical approximation does not exploit the optical theorem, but instead combines a simple translation of the computational coordinate into the complex 
plane with a rational Chebyshev series and the QR algorithm . The shifted/Chebyshev/QR algorithm is easy to program but is limited by roundoff error to computing values of $\Im(\lambda)$ that are no smaller than the machine precision multiplied by the real part of $\lambda$.

The second numerical scheme combines the optical theorem with perturbative estimates for the eigenvalue and an exponential change of coordinate to compute the eigenvalue through a non-iterative initial value algorithm. It has the great virtue that it can compute extremely small values of $\Im(\lambda)$. Although very tiny $\Im(\lambda)$ are not useful in any practical engineering application, they are valuable in checking the analytical approximations.

2. The Optical Theorem and the Non-Uniformity of the Zero Friction Limit. Theorem 2.1 (Optical Relation). Suppose the ODE is

$$
u_{z z}+\left\{\frac{r(z)}{z-i \delta(z)}+p(z)-\lambda\right\} u=0
$$

on the interval $z \in[A, B]$ where $r(z)>0$. Then

$$
\Im(\lambda) \int_{A}^{B}|u(z)|^{2} d z=\int_{A}^{B} \frac{\delta(z)}{z^{2}+\delta^{2}(z)} r(z)|u(z)|^{2} d z
$$

Special Cases:

1. If the friction $\delta$ is a constant, then in the limit $\delta \rightarrow 0$

$$
\Im(\lambda)=\pi r(0) \frac{|u(0)|^{2}}{\int_{A}^{B}|u(z)|^{2} d z}
$$

2. If the friction is piecewise-constant, centered on the singularity,

$$
\delta(z)=\left\{\begin{array}{c}
0, \quad|z|>\omega \\
\nu, \quad z \in[-\omega, \omega]
\end{array}\right.
$$

then in the limit $\nu, \omega \rightarrow 0$ for fixed ratio of $\omega / \nu$

$$
\Im(\lambda)=2 \arctan \left\{\frac{\omega}{\nu}\right\} r(0) \frac{|u(0)|^{2}}{\int_{A}^{B}|u(z)|^{2} d z}
$$

Proof: The general case is Eq.(2.12) of [7]. The first special case is Theorem 2 of [7] (with correction of a typographical error). The second special case follows from expanding the $\delta$-independent parts of the integrand about the singularity, keeping just the constants, and pulling the constants out of the integrand. The remaining integrand can then be analytically integrated to give the arctangent.

In the rest of this article, we shall take only the uniform limit. (The same eigenvalue is obtained by setting the friction equal to zero and solving the differential equation on a contour that is deformed slightly into the lower half of the complex plane.) Eq.(7) is the magic key that unchains this eigenproblem from its technical difficulties. It is no longer necessary to calculate $\Im(\lambda)$ directly as the exponentially small part of a much larger eigenvalue. Instead, one merely needs the magnitude of the normalized eigenfunction at the critical latitude.

It has been previously assumed that the limit of zero friction was uniform so that one would obtain the same $\Im(\lambda)$ no matter how the limit was taken. The second special case shows that this assumption is false. If the friction is non-zero only in a layer which is thin compared to the strength of the friction, then $\Im(\lambda)$ can be very small in magnitude compared to its magnitude in the different limit $\delta \rightarrow 0$ when $\delta$ is independent of $z$. 
3. Rayleigh-Schrödinger Perturbation Theory. In terms of the shifted coordinate

$$
y \equiv z-1 / \epsilon, \quad \leftrightarrow \quad z=y+1 / \epsilon
$$

where $y=0$ is the equator, the Hermite-with-Pole equation is

$$
u_{y y}+\left\{\epsilon \frac{1}{1+\epsilon y}-y^{2}\right\} u=\lambda u
$$

The unperturbed $(\epsilon=0)$ eigenfunctions are the unnormalized Hermite functions defined by the recurrence relation

$$
\begin{gathered}
\psi_{0} \equiv \exp \left(-(1 / 2) y^{2}\right) \quad ; \quad \psi_{1}(y) \equiv 2 y \psi_{0} \\
\psi_{n+1}(y)=2 y \psi_{n}(y)-2 n \psi_{n-1}(y)
\end{gathered}
$$

The eigenvalue and eigenfunctions for nonzero $\epsilon$ can be computed by expanding both as well as the coefficients of the differential equation in powers of $\epsilon$ and matching terms order-by-order. Here, as in the rest of the article, we shall compute only the ground state, which is $\psi_{0}(y)$ in the limit $\epsilon \rightarrow 0$, but the method is applicable to the higher eigenfunctions, too. The perturbation series is identical, except for the need to expand $1 /(1+\epsilon y)$, to the usual Rayleigh-Schrödinger expansion for computing bound states in quantum mechanics. Rather than rehash the details, Table 1 offers instead the complete Maple program for computing the series to arbitrary order.

The eigenvalue series is

$$
\begin{aligned}
\lambda= & -1+\epsilon+\frac{1}{2} \epsilon^{3}+\frac{1}{4} \epsilon^{4}+\frac{3}{4} \epsilon^{5}+\frac{7}{8} \epsilon^{6}+\frac{17}{8} \epsilon^{7}+\frac{53}{16} \epsilon^{8}+\frac{17}{2} \epsilon^{9}+\frac{121}{8} \epsilon^{10} \\
& +\frac{1347}{32} \epsilon^{11}+\frac{10511}{128} \epsilon^{12}+\frac{7873}{32} \epsilon^{13}+\frac{66171}{128} \epsilon^{14}+\frac{424213}{256} \epsilon^{15}+\ldots
\end{aligned}
$$

The eigenfunction at each order is a finite sum of Hermite functions whose coefficients are given in Table 2 where the $a_{j k}$ are defined by

$$
u(y)=\sum_{j} \epsilon^{j} \sum_{k=0}^{\infty} a_{j k} \psi_{k}(y)
$$

For use with the optical theorem, note that if the eigenfunction is rescaled by dividing by $u(y=0 ; \epsilon)$ so that the rescaled eigenfunction has $\tilde{u}(y=0 ; \epsilon)=1$, then the Rayleigh-Schrödinger series shows

$$
\int_{-\infty}^{\infty}|\tilde{u}(y, \epsilon)|^{2} d y=\sqrt{\pi}\left(1+(1 / 4) \epsilon^{3}+(1 / 4) \epsilon^{4}+\ldots\right)
$$

The rapid growth of the eigenvalue coefficients suggests that the series is asymptotic but divergent. In Appendix A, we offer a comparison with the series for a related integral to argue heuristically that the coefficients $\lambda_{j}$ are growing as roughly the square root of $j$ !. The divergence is not surprising because the Hermite-with-Pole problem is posed on an infinite interval, but the geometric series for the perturbation $1 /(1+\epsilon y)$ only converges for $|y|<1 / \epsilon$. However, at the limit of convergence, the ground state eigenfunction has a magnitude of roughly $\exp \left(-(1 / 2) / \epsilon^{2}\right)$ as justified by evaluating the unperturbed eigenfunction at $y=1 / \epsilon$. It follows that the geometric 
TABLE 1

Rayleigh-Schrödinger Maple program

\begin{tabular}{|c|}
\hline 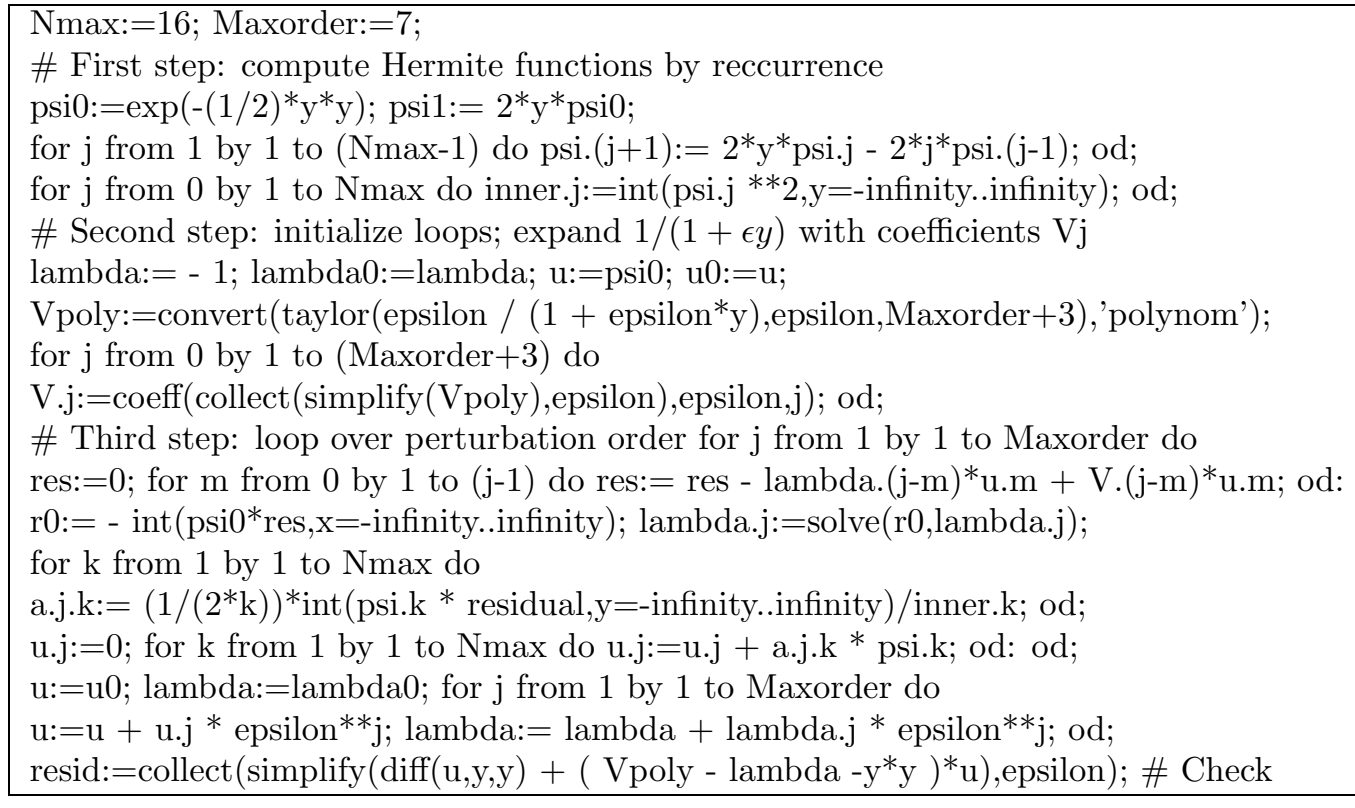 \\
\hline
\end{tabular}

TABLE 2

Rayleigh-Schroedinger Series for $u_{z z}+\left(1 / z-\lambda-(z-1 / \epsilon)^{2}\right) u=0$ : Hermite Coefficients

\begin{tabular}{|l|l|l|l|l|l|l|l|}
\hline$j$ & $a_{j 0}$ & $a_{j 1}$ & $a_{j 2}$ & $a_{j 3}$ & $a_{j 4}$ & $a_{j 5}$ & $a_{j 6}$ \\
\hline$\epsilon^{0}$ & 1 & 0 & 0 & 0 & 0 & 0 & 0 \\
\hline$\epsilon$ & 0 & 0 & 0 & 0 & 0 & 0 & 0 \\
\hline$\epsilon^{2}$ & 0 & $-\frac{1}{4}$ & 0 & 0 & 0 & 0 & 0 \\
\hline$\epsilon^{3}$ & 0 & 0 & $\frac{1}{16}$ & 0 & 0 & 0 & 0 \\
\hline$\epsilon^{4}$ & 0 & $-\frac{3}{8}$ & $\frac{1}{32}$ & $-\frac{1}{48}$ & 0 & 0 & 0 \\
\hline$\epsilon^{5}$ & 0 & $-\frac{3}{16}$ & $\frac{3}{16}$ & $-\frac{1}{64}$ & $\frac{1}{128}$ & 0 & 0 \\
\hline$\epsilon^{6}$ & 0 & $-\frac{15}{16}$ & $\frac{3}{16}$ & $-\frac{41}{384}$ & $\frac{11}{1536}$ & $-\frac{1}{320}$ & 0 \\
\hline$\epsilon^{7}$ & 0 & -1 & $\frac{3}{4}$ & $-\frac{47}{384}$ & $\frac{31}{512}$ & $-\frac{5}{1536}$ & $\frac{1}{768}$ \\
\hline
\end{tabular}

series diverges only where the amplitude of the eigenfunction is exponentially small. The errors we make by pushing the series beyond its radius of convergence will be exponentially small in $1 / \epsilon^{2}$. If the series is "optimally truncated" by adding terms up to and including the smallest term for a given $\epsilon$, then the expected error will be $O\left(\exp \left(-(1 / 2) / \epsilon^{2}\right)\right.$.

Berry[4] has dubbed such an optimally-truncated series a "superasymptotic" approximation. His reason for introducing new terminology is that although the usual Poincaré definition of asymptoticity refers only to powers of $\epsilon$, the error in an optimally-truncated series is usually, as here, an exponential function of $1 / \epsilon$.

Unfortunately, this expected error is the same order of magnitude as the imaginary part of the eigenvalue! It follows that to compute $\Im(\lambda)$, we need something better: the matched asymptotics analysis of the next three sections. 
4. Middle Region Analysis: Between the Critical Latitude and Equator. The solution in the middle region could be approximated by a standard WKB expansion (Bender, Orszag, 1978). However, this involves a messy phase integral. Furthermore, the truncated Rayleigh-Schrödinger series can be represented as

$$
u=\exp \left(-\frac{y^{2}}{2}\right) P(y ; \epsilon)
$$

where $P(y ; \epsilon)$ is a known polynomial in $y$ and $\epsilon$. It is therefore convenient to replace WKB by an approximation of the form

$$
u=\exp \left(-\frac{1}{2} \frac{Y^{2}}{\epsilon^{2}}\right) v(Y)
$$

where

$$
Y \equiv \epsilon y \quad \leftrightarrow \quad y \equiv Y / \epsilon
$$

The Hermite-with-Pole equation is transformed to

$$
v_{Y Y}-2 \frac{Y}{\epsilon^{2}} v_{Y}+\left(-\frac{Y}{\epsilon(1+Y)}-\frac{\tilde{\lambda}}{\epsilon^{2}}\right) v=0
$$

where

$$
\tilde{\lambda}=\lambda-(-1+\epsilon) \sim \frac{1}{2} \epsilon^{3}+\frac{1}{4} \epsilon^{4}+\frac{1}{4} \epsilon^{5}+\ldots
$$

If we expand $v$ as the power series in $\epsilon$

$$
v(Y)=1+\epsilon v_{1}(Y)+\epsilon^{2} v_{2}(Y)+\ldots,
$$

each $v_{j}$ satisfies an equation of the form

$$
v_{j, Y}=f_{j}(Y) \Rightarrow v_{j}=b_{j}+\int^{Y} f_{j}\left(Y^{\prime}\right) d Y^{\prime} \quad j=1,2, \ldots
$$

where $f_{j}$ depends only on previously computed terms and $b_{j}$ are constants of integration, which can later be chosen to ensure a perfect match with the RayleighSchrödinger series. It turns out that for the first order matching we only need terms up to $v_{3}(Y)$ :

$$
\begin{array}{r}
v \sim 1+\epsilon\left[b_{1}-\frac{1}{2} \log (1+Y)\right] \\
+\epsilon^{2}\left[b_{2}+\frac{1}{8} \log ^{2}(1+Y)\right] \\
+\epsilon^{3}\left[b_{3}+\frac{1}{4} \frac{1}{1+Y}-\frac{1}{4} \log (1+Y)-\frac{1}{48} \log ^{3}(1+Y)\right] \\
+O\left(\epsilon^{4}\right)
\end{array}
$$

or in terms of $y$

$$
v \sim 1+\epsilon\left[b_{1}-\frac{1}{2} \log (1+\epsilon y)\right]
$$




$$
\begin{array}{r}
+\epsilon^{2}\left[b_{2}+\frac{1}{8} \log ^{2}(1+\epsilon y)\right] \\
+\epsilon^{3}\left[b_{3}+\frac{1}{4} \frac{1}{1+\epsilon y}-\frac{1}{4} \log (1+\epsilon y)-\frac{1}{48} \log ^{3}(1+\epsilon y)\right] \\
+O\left(\epsilon^{4}\right)
\end{array}
$$

Now we Taylor-expand and rearrange this:

$$
\begin{array}{r}
v \sim 1+\epsilon b_{1}+\epsilon^{2}\left[-\frac{1}{2} y+b_{2}\right] \\
+\epsilon^{3}\left[-\frac{1}{2} y^{2}+b_{3}\right]+O\left(\epsilon^{4}\right)
\end{array}
$$

Eq. (23) matches $P(y ; \epsilon)$ if and only if

$$
\begin{array}{r}
b_{1}=0 \\
b_{2}=0 \\
b_{3}=-\frac{3}{8}
\end{array}
$$

After substitution of (24) into (21), we obtain

$$
\begin{array}{r}
v \sim 1+\epsilon\left[-\frac{1}{2} \log (1+Y)\right] \\
+\epsilon^{2}\left[\frac{1}{8} \log ^{2}(1+Y)\right] \\
+\epsilon^{3}\left[-\frac{3}{8}+\frac{1}{4} \frac{1}{1+Y}-\frac{1}{4} \log (1+Y)-\frac{1}{48} \log ^{3}(1+Y)\right] \\
+O\left(\epsilon^{4}\right)
\end{array}
$$

This is not normalized to a value of unity at $Y=0$, but rather has the same normalization as the Rayleigh-Schrödinger series.

Next step is to express this in terms of the natural variable of the near-the-pole region:

$$
\zeta \equiv z / \epsilon \quad \leftrightarrow \quad z \equiv \epsilon \zeta
$$

We transform the middle-region series to the Whittaker region by replacing $Y$ by $\zeta$

$$
\begin{gathered}
Y=\epsilon(z-1 / \epsilon) \\
u \sim \exp \left(-\frac{1}{2 \epsilon^{2}}+\zeta-\frac{1}{2} \epsilon^{2} \zeta^{2}\right)\left\{1-\frac{\epsilon}{2} \log \left(\epsilon^{2} \zeta\right)+\frac{\epsilon^{2}}{8} \log ^{2}\left(\epsilon^{2} \zeta\right)\right. \\
\left.+\epsilon^{3}\left(-\frac{3}{8}+\frac{1}{4} \frac{1}{\epsilon^{2} \zeta}-\frac{1}{4} \log \left(\epsilon^{2} \zeta\right)-\frac{1}{48} \log ^{3}\left(\epsilon^{2} \zeta\right)\right)+\ldots\right\}
\end{gathered}
$$

For $\zeta<\frac{1}{\sqrt{\epsilon}}$, it is consistent to reexpand this for fixed $\zeta$ as

$$
u \sim \exp \left(-\frac{1}{2 \epsilon^{2}}+\zeta\right)\left\{1+\epsilon\left(-\frac{1}{2} \log \zeta-\log \epsilon+\frac{1}{4 \zeta}+O\left(\frac{1}{\zeta^{2}}\right)\right)+\ldots\right\}
$$


(Contributions of order $O\left(\frac{1}{\zeta^{2}}\right)$ come from the terms of order $O\left(\epsilon^{5}\right)$ and higher in $(25)$, and thus are not written explicitly in 27.) This reexpansion should match the local approximation near the pole which is derived in the next section.

5. Approximations Near the Pole. After the change of the independent variable (26), the Hermite-with-Pole equation transforms to

$$
u_{\zeta \zeta}+\left(\frac{\epsilon}{\zeta}-1-\lambda \epsilon^{2}+2 \epsilon^{2} \zeta-\epsilon^{4} \zeta^{2}\right) u=0
$$

We split $Q(\zeta)=\frac{\epsilon}{\zeta}-1-\lambda \epsilon^{2}+2 \epsilon^{2} \zeta-\epsilon^{4} \zeta^{2}$ into two parts $\rho(\zeta)$ and $\delta(\zeta)$, where

$$
\rho(\zeta)=\frac{\epsilon}{\zeta}-1
$$

is the dominant part of $Q(\zeta)$ and

$$
\delta(\zeta)=-\lambda \epsilon^{2}+2 \epsilon^{2} \zeta-\epsilon^{4} \zeta^{2}
$$

is a small perturbation. For the unperturbed equation

$$
u_{\zeta \zeta}+\rho(\zeta) u=0
$$

the solution that satisfies the condition $u(\zeta) \rightarrow 0$ as $\zeta \rightarrow-\infty$ is the Whittaker function

$$
C \Gamma\left(1+\frac{\epsilon}{2}\right) W_{-\frac{\epsilon}{2}, \frac{1}{2}}(-2 \zeta)
$$

where $C$ is a constant and $u(\zeta=0)=C$ as shown on p.1588 of [7]. For matching, we only need terms up to $O(\epsilon)$ in the solution of the perturbed problem (28). But Eq. (28) can be rewritten as

$$
u_{\zeta \zeta}+\rho(\zeta) u=-\delta(\zeta) u=O\left(\epsilon^{2}, \epsilon^{2} \zeta, \epsilon^{4} \zeta^{2}\right)
$$

The right hand side of this expression is $o(\epsilon)$ if $\zeta<1 / \epsilon$. This implies that (32) solves (28) within $o(\epsilon)$ for $\zeta<1 / \epsilon$.

\section{Asymptotic Matching of the Whittaker Function to Middle Region} Expansion. Now we need the asymptotic approximation of (32) for large $\zeta$. Note that $W_{-\frac{\epsilon}{2}, \frac{1}{2}}(-2 \zeta)$ has a branch point at $\zeta=0$. The simplest physically allowable branch cut lies along the ray

$$
\arg (-2 \zeta)=-\pi / 2
$$

as explained on p.1578 of [7]. Formula (4.2) of that paper gives the asymptotic expansions for Whittaker functions for this nonstandard choice of branch:

$$
\begin{array}{r}
\Gamma\left(1+\frac{\epsilon}{2}\right) W_{-\frac{\epsilon}{2}, \frac{1}{2}}(-2 \zeta)=\frac{\Gamma\left(1+\frac{\epsilon}{2}\right) \exp (\zeta)}{(-2 \zeta)^{\epsilon / 2}}\left(1-\frac{\epsilon}{2} \frac{\left(1+\frac{\epsilon}{2}\right)}{-2 \zeta}+\frac{\epsilon}{2} \frac{\left(1+\frac{\epsilon}{2}\right)\left(1+\frac{\epsilon}{2}\right)\left(2+\frac{\epsilon}{2}\right)}{(-2 \zeta)^{2}}+\ldots\right) \\
\approx\left(1-\frac{\gamma}{2} \epsilon\right) \frac{\exp (\zeta)}{(-2 \zeta)^{\epsilon / 2}}\left(1+\frac{\epsilon}{\zeta}+O\left(\frac{\epsilon}{\zeta^{2}}\right)\right)
\end{array}
$$

In (35) we used the fact that

$$
\Gamma\left(1+\frac{\epsilon}{2}\right) \approx 1-\frac{\gamma}{2} \epsilon+O\left(\epsilon^{2}\right)
$$


where $\gamma \approx 0.577$ is the Euler-Macheroni constant. Finally, after approximating

$$
\frac{1}{(-2 \zeta)^{\epsilon / 2}} \approx 1-\frac{\epsilon}{2}\{\log \zeta+(-\log 2+i \pi)\}
$$

we obtain the following expansion of (32) for $\zeta \gg 1$ (to the first order in $\epsilon$ )

$$
u \approx C\left(1-\frac{\gamma}{2} \epsilon\right) \exp (\zeta)\left\{1+\epsilon\left(-\frac{1}{2} \log \zeta-\frac{\log 2-\pi i}{2}+\frac{1}{4 \zeta}+O\left(\frac{1}{\zeta^{2}}\right)\right)+\ldots\right\}
$$

This is of the same form as the middle region approximation (27). Both (27) and (38) are accurate within $o(\epsilon)$ in the overlap region $1 \ll \zeta<\frac{1}{\sqrt{\epsilon}}$ and

$$
C=\left\{1+\epsilon \frac{\log 2-\pi i}{2}-\epsilon \log \epsilon+\epsilon \frac{\gamma}{2}\right\} \exp \left(-\frac{1}{2 \epsilon^{2}}\right)=\tilde{u}(0)
$$

Now we can substitute this result into the optical theorem to find

$$
\Im(\lambda)=\pi \frac{|\tilde{u}(0)|^{2}}{\int_{-\infty}^{\infty}|\tilde{u}|^{2} d z}=\sqrt{\pi} \exp \left(-\frac{1}{\epsilon^{2}}\right)\{1-2 \epsilon \log \epsilon+\epsilon \log 2+\gamma \epsilon\}
$$

7. Numerical Method I: Chebyshev Pseudospectral/QR algorithm with a Shift into the Complex Plane. Our first numerical algorithm is a Chebyshev pseudospectral algorithm. The solution is expanded as a series of the rational Chebyshev functions $T B_{j}[8,10]$, the expansion (truncated after $N$ terms) is substituted into the differential equation to define the "residual" function, and the series coefficients are chosen so that the residual is zero at each of $N$ collocation points. These conditions convert the differential eigenproblem into a matrix generalized eigenproblem which can be solved by the QR/QZ algorithm. (We used the eigensolver which is built into the language/software package MATLAB.) Although the QR algorithm is relatively expensive $\left(O\left(10 N^{3}\right)\right.$ operations), this procedure is very robust and does not require a first guess for any eigenvalues.

Unfortunately, the eigenfunction is singular. This implies that the spectral series will converge very slowly. Boyd[9] proposed a remedy: making a change of coordinate to a new variable $w$ such that real-valued $w$ corresponds to a curve in the complex $z$-plane which detours a safe distance below the singularity in the lower half of the complex $z$-plane. As shown by Boyd and Gill and Sneddon[14, 15], this trick is effective; mappings can be devised for any almost combination of boundaries and critical latitudes.

Here, however, a simple shift of the contour of integration by an imaginary constant will suffice:

$$
w(z) \equiv z-1 / \epsilon+i \sigma \quad \Leftrightarrow \quad z=1 / \epsilon+w-i \sigma
$$

where $\sigma>0$ is a real constant. The real interval $w \in[-\infty, \infty]$ is a line parallel to the real $z$-axis at $\Im(z)=-\sigma$ as illustrated in Fig. 1. Since $u(z(w))$ is not singular for any real $w$, the rational Chebyshev series converges with spectral accuracy[11, 9, 10].

The basis functions are

$$
T B_{j}(w ; L) \equiv \cos (j t) ; \quad t(w) \equiv \operatorname{arccot}(w / L)
$$




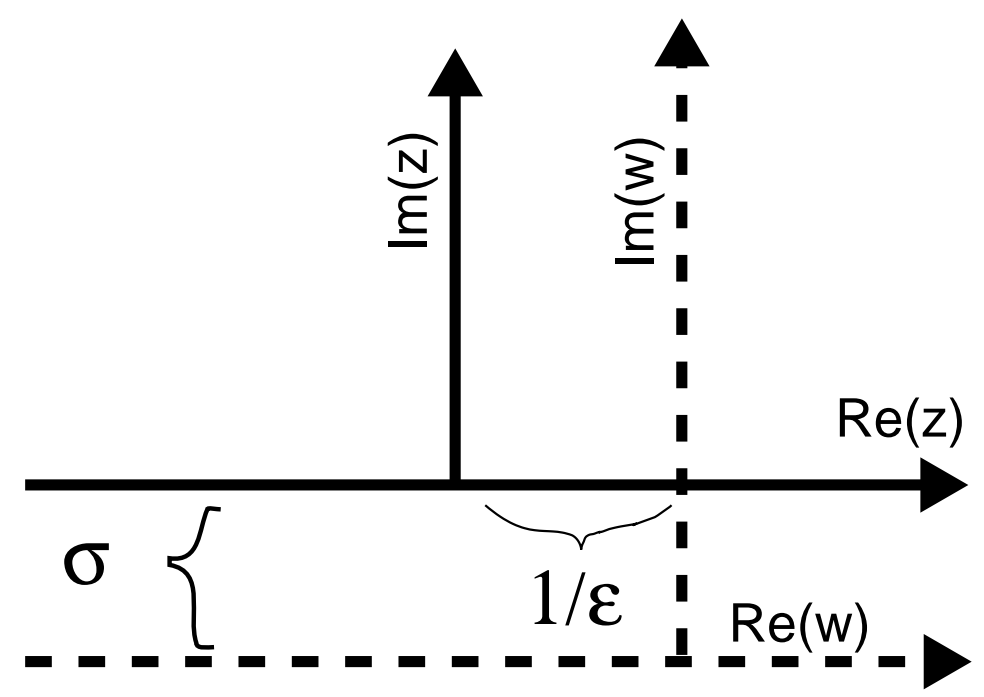

FIG. 1. Schematic of the complex $z$-plane with the real and imaginary axes for the new shifted coordinate, with the Chebyshev/QR method, shown as the dashed lines. The coordinates are shifted by $\sigma$ in the imaginary direction and by $1 / \epsilon$ in the real axis. The differential equation is singular at $z=0$; the equator is $z=1 / \epsilon$.

and the matrix eigenproblem $H a=\lambda B a$ has the elements

$$
\begin{aligned}
H_{i j} & \equiv T B_{j, w w}\left(w_{i} ; L\right)+\left(1 /\left(1 / \epsilon+w_{i}-i \sigma\right)-\left(w_{i}-i \sigma\right)^{2}\right) T B_{j}\left(w_{i} ; L\right) \\
B_{i j} & \equiv T B_{j}\left(w_{i} ; L\right)
\end{aligned}
$$

where $a$ is a column vector containing the $N$ coefficients of the truncated $T B$ series, $L$ is a user-choosable constant ("map parameter") and the collocation points $w_{j}$ are

$$
w_{i}=L \cot \left(\frac{(2 i-1) \pi}{2 N}\right), \quad i=1,2, \ldots, N
$$

and where derivatives of the basis functions are calculated by making the change of variable to the trigonometric coordinate $t$ and then applying the chain rule.

For each $\epsilon$ and a given choice of $L$ and $\sigma$, it is necessary to repeat the calculation for increasing $N$ until the eigenvalue becomes approximately independent of $N$ as illustrated in Table 3. Different values of $L$ and $\sigma$ were also used to confirm that the eigenvalues are independent of these map parameters for sufficiently large $N$.

Although the rational Chebyshev method is a robust and extremely accurate method for moderate $\epsilon$, the spread in eigenvalues for $\epsilon=1 / 5$ shows that for this and smaller $\epsilon, \Im(\lambda)$ is so tiny compared to the real part of $\lambda$ that roundoff errors corrupt $\Im(\lambda)$. Furthermore, since the spectral series converges rapidly only on a line in the complex plane, it is not useful for graphing the eigenfunction for real $z$. We therefore developed a shooting method as described in the next section.

8. Numerical Method II: Shooting with Exponential Mapping. The shooting algorithm exploits both the optical theorem and the Rayleigh-Schrödinger series. The theorem implies that to determine the imaginary part of $\lambda$, it is sufficient to calculate the value of $|\tilde{u}(0)|$ where $\tilde{u}$ denotes an eigenfunction that has been normalized 
TABLE 3

Lowest Eigenvalue $\lambda$ of $u_{z z}+\left(1 / z-\lambda-(z-1 / \epsilon)^{2}\right) u=0$ : Rational Chebyshev Computations

\begin{tabular}{|l|l|l|l|l|l|}
\hline$\epsilon$ & $L$ & $\sigma$ & $N$ & $\Re(\lambda)$ & $\Im(\lambda)$ \\
\hline 1 & 1 & 1 & 50 & -0.13928394 & 0.630023033 \\
\hline 1 & 1 & 1 & 100 & -0.13928433 & 0.630023042 \\
\hline
\end{tabular}

\begin{tabular}{|l|l|l|l|l|l|}
\hline $3 / 4$ & 1 & 1 & 70 & -0.1672609872 & 0.38677068511 \\
\hline $3 / 4$ & 1 & 1 & 100 & -0.1672609845 & 0.38677068607 \\
\hline
\end{tabular}

\begin{tabular}{|l|l|l|l|l|l|}
\hline $1 / 2$ & 1 & 1 & 50 & -0.3763763143824 & 0.07805050579007 \\
\hline $1 / 2$ & 1 & 1 & 100 & -0.3763768356309 & 0.07805030263300 \\
\hline $1 / 2$ & 1 & 1 & 150 & -0.3763768356281 & 0.07805030263281 \\
\hline $1 / 2$ & 2 & 1 & 200 & -0.3763768356290 & 0.07805030263097 \\
\hline
\end{tabular}

\begin{tabular}{|l|l|l|l|l|l|}
\hline $2 / 5$ & 1 & 1 & 70 & -0.53789788669694 & 0.01023260239293 \\
\hline $2 / 5$ & 1 & 1 & 100 & -0.53789788277539 & 0.01023260166942 \\
\hline $2 / 5$ & 2 & 1 & 200 & -0.53789788277396 & 0.01023260166725 \\
\hline
\end{tabular}

\begin{tabular}{|l|l|l|l|l|l|}
\hline $1 / 3$ & 1 & 1 & 50 & -0.6375758962641 & 0.0006592594408 \\
\hline $1 / 3$ & 1 & 1 & 100 & -0.6375763991223 & 0.0006593424970 \\
\hline $1 / 3$ & 1 & 1 & 150 & -0.6375763991223 & 0.0006593424787 \\
\hline $1 / 3$ & 1 & 2 & 50 & -0.63719537711198 & 0.00074355861757 \\
\hline $1 / 3$ & 1 & 2 & 200 & -0.63757639911178 & 0.00065934248393 \\
\hline
\end{tabular}

\begin{tabular}{|l|l|l|l|l|l|}
\hline $1 / 4$ & 1 & 1 & 100 & -0.74001184495767 & 0.00000053832889 \\
\hline $1 / 4$ & 1 & 1 & 150 & -0.74001184496886 & 0.00000053830828 \\
\hline
\end{tabular}

\begin{tabular}{|l|l|l|l|l|l|}
\hline $1 / 5$ & 1 & 1 & 100 & -0.79526081693861 & 0.00000000006614 \\
\hline $1 / 5$ & 1 & 1 & 200 & -0.79526081694896 & 0.00000000006055 \\
\hline $1 / 5$ & 1 & 1 & 300 & -0.79526081695029 & 0.00000000005887 \\
\hline $1 / 5$ & 2 & 1 & 200 & -0.79526081694884 & 0.00000000006099 \\
\hline $1 / 5$ & 1 & $1 / 2$ & 200 & -0.79526081694952 & 0.00000000006044 \\
\hline
\end{tabular}

so that $\tilde{u}(1 / \epsilon)=1$. The divergent Rayleigh-Schrödinger series is useful in two ways. First, when truncated at the smallest term, it provides an approximation to the real part of the eigenvalue which is accurate to $O\left(\exp \left(-1 / \epsilon^{2}\right)\right)$. Usually it is necessary to iterate to compute the eigenvalue simultaneously with the eigenfunction. Here, however, the Rayleigh-Schrödinger series gives the eigenvalue to sufficient accuracy so that it is unnecessary to iterate. The exponentially small error of the series includes the entire imaginary part of $\lambda$, but we shall obtain $\Im(\lambda)$ from the optical theorem; for computing the eigenfunction, it is sufficient to know only the (much larger) real part of $\lambda$. The second usefulness of the Rayleigh-Schrödinger series is that it gives the integral of $|\tilde{u}|^{2}$ over the whole domain to high accuracy as Eq.(16).

Thus, to compute $\Im(\lambda)$, it is unnecessary to iterate or to evaluate the normalization integral. Instead, one merely needs to integrate the differential equation once. We employed the fourth-order Runge-Kutta algorithm. There are two remaining technical complications. 
The first is that the differential equation is very "stiff" in the sense that it has growing and decaying solutions which vary by many orders of magnitude over the domain. The remedy is to simply march always in the direction in which the desired solution is growing. The eigenfunction decays exponentially as $z \rightarrow-\infty$ for $z<0$. Therefore, we integrate for negative $z$ by marching from large negative $z$ towards the singularity at $z=0$. Arbitrary initial conditions at $z=-L$ where $L$ is a userchoosable constant will excite both the growing and decaying solutions. However, as we march towards the singularity, the growing solution rapidly becomes exponentially large compared to the decaying solution so that the numerical solution becomes what we want: the solution which grows as $z \rightarrow 0^{-}$. Between $z=0$ and $z=1 / \epsilon$, the solution also grows as $z$ increases, so the stiffness is again no problem as long as we march in the direction of increasing $z$.

The second technical complication is that the differential equation is singular at $z=0$. This difficulty is resolved in two steps. The first is to stop the integration for small negative $z$ where the power series approximation

$$
u(z ; \epsilon) \approx 1-z \log (z)+a_{1} z+O\left(z^{2} \log (z)\right)
$$

is accurate, and then restart the integration at small positive $z$ using the power series to supply the initial conditions for the second round of Runge-Kutta. The continuation across the singularity is non-trivial in a couple of ways. First, we found it helpful to calculate $a_{1}$ by fitting the numerical results for very small $z$; technically, one could ignore it for sufficiently small $z$, but the logarithmic term dominates the linear term only when $z$ is very tiny. Second, the logarithm must be interpreted as

$$
\log (z)= \begin{cases}\log (|z|), & z<0 \\ \log (|z|)-\pi i, & z>0\end{cases}
$$

This is necessary so that the branch cut is taken to lie in the upper half-plane, a choice motivated by physics [7]rather than mathematics.

The second remedy for the singularity is to use an exponential mapping so that an even grid spacing in the new coordinate corresponds to an exponentially high density of grid points in the vicinity of $z=0$ where the singularity requires very high numerical resolution. The mapping to the left of the singularity is

$$
x \equiv-\log (-z / L) \Leftrightarrow z=-L \exp (-x) \quad x \in[0, \infty], z \in[-L, 0],
$$

The chain rule shows that derivatives transform as

$$
\frac{d}{d z}=\frac{\exp (x)}{L} \frac{d}{d x}, \quad \frac{d^{2}}{d z^{2}}=\frac{\exp (2 x)}{L^{2}}\left\{\frac{d^{2}}{d x^{2}}+\frac{d}{d x}\right\}
$$

We then march from $x=0$, which corresponds to $z=-L$, to large but finite $x$. Since the singularity is at $x=\infty$, the Runge-Kutta integration never reaches the singular point and is always stable and accurate. The constant $L$ is arbitrary except that it should be chosen so that the solution grows by many orders of magnitude on the interval $z \in[-L, 0]$.

The appropriate coordinate change on the right of the singularity is, with $M \equiv 1 / \epsilon$

$$
\begin{gathered}
x \equiv-\log (z / M) \Leftrightarrow z=M \exp (-x) \quad x \in[0, \infty], z \in[0, M] \\
\frac{d}{d z}=-\frac{\exp (x)}{M} \frac{d}{d x}, \quad \frac{d^{2}}{d z^{2}}=\frac{\exp (2 x)}{M^{2}}\left\{\frac{d^{2}}{d x^{2}}+\frac{d}{d x}\right\}
\end{gathered}
$$




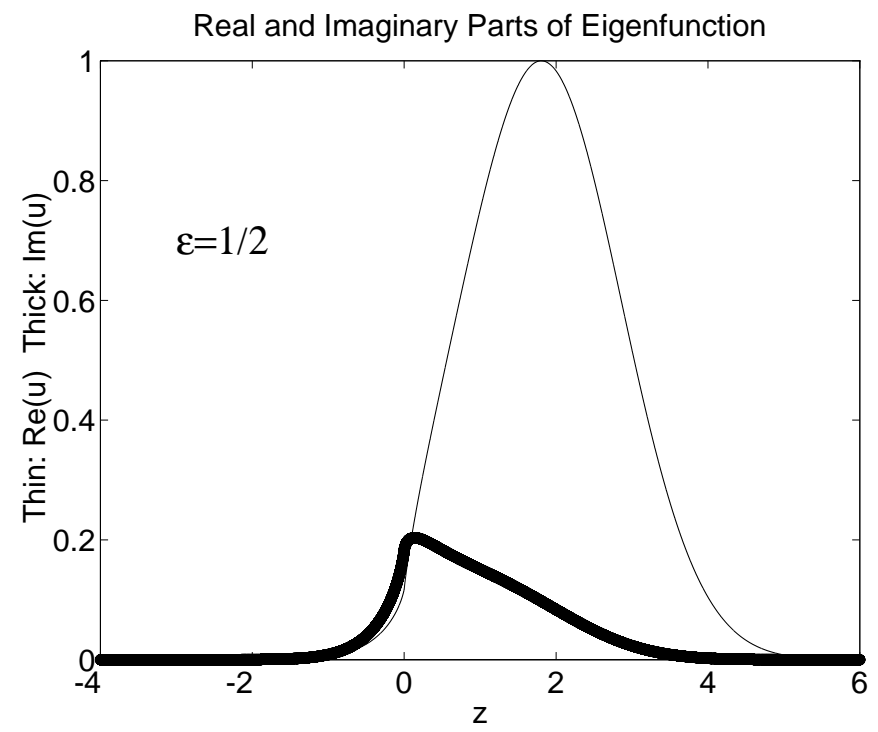

FIG. 2. Real and imaginary parts of the eigenfunction for $\epsilon=1 / 2$. (The thin curve is $\Re(u)$; the thick curve is the imaginary part.

It is necessary to use larger and larger numbers of points as $\epsilon \rightarrow$. However, as judged by comparing solutions for different choices of $L$, the maximum value of the computational coordinate $x$, and the grid spacing,we did obtain satisfactory results for $\epsilon$ as small as $1 / 100$.

Because the initial value algorithm does not use a shift in the complex $z$-plane, it accurately computes the eigenfunction for real $z$ as illustrated in Fig. 2.

9. Comparisons Between Numerical Solutions and Matched Asymptotics. In this section we compare approximations

$$
\begin{gathered}
\Im(\lambda)_{0}=\sqrt{\pi} \exp \left(-\frac{1}{\epsilon^{2}}\right) \\
\Im(\lambda)_{1}=\sqrt{\pi} \exp \left(-\frac{1}{\epsilon^{2}}\right)\{1-2 \epsilon \log \epsilon\} \\
\Im(\lambda)_{2}=\sqrt{\pi} \exp \left(-\frac{1}{\epsilon^{2}}\right)\{1-2 \epsilon \log \epsilon+(\log 2+\gamma) \epsilon\}
\end{gathered}
$$

to the numerical results $\Im(\lambda)_{\text {Num }}$.

We define the relative error of a perturbative approximation $\Im(\lambda)_{i}$ as

$$
E_{i}=|| \frac{\Im(\lambda)_{N u m}}{\Im(\lambda)_{i}}|-1|
$$

Fig. 3 confirms that the relative error does become smaller as we add more terms to the perturbative approximation. The surprising part is that the relative error of the zeroth-order approximation is of order 10 per cent for $\epsilon$ as small as $1 / 100$ ! This 


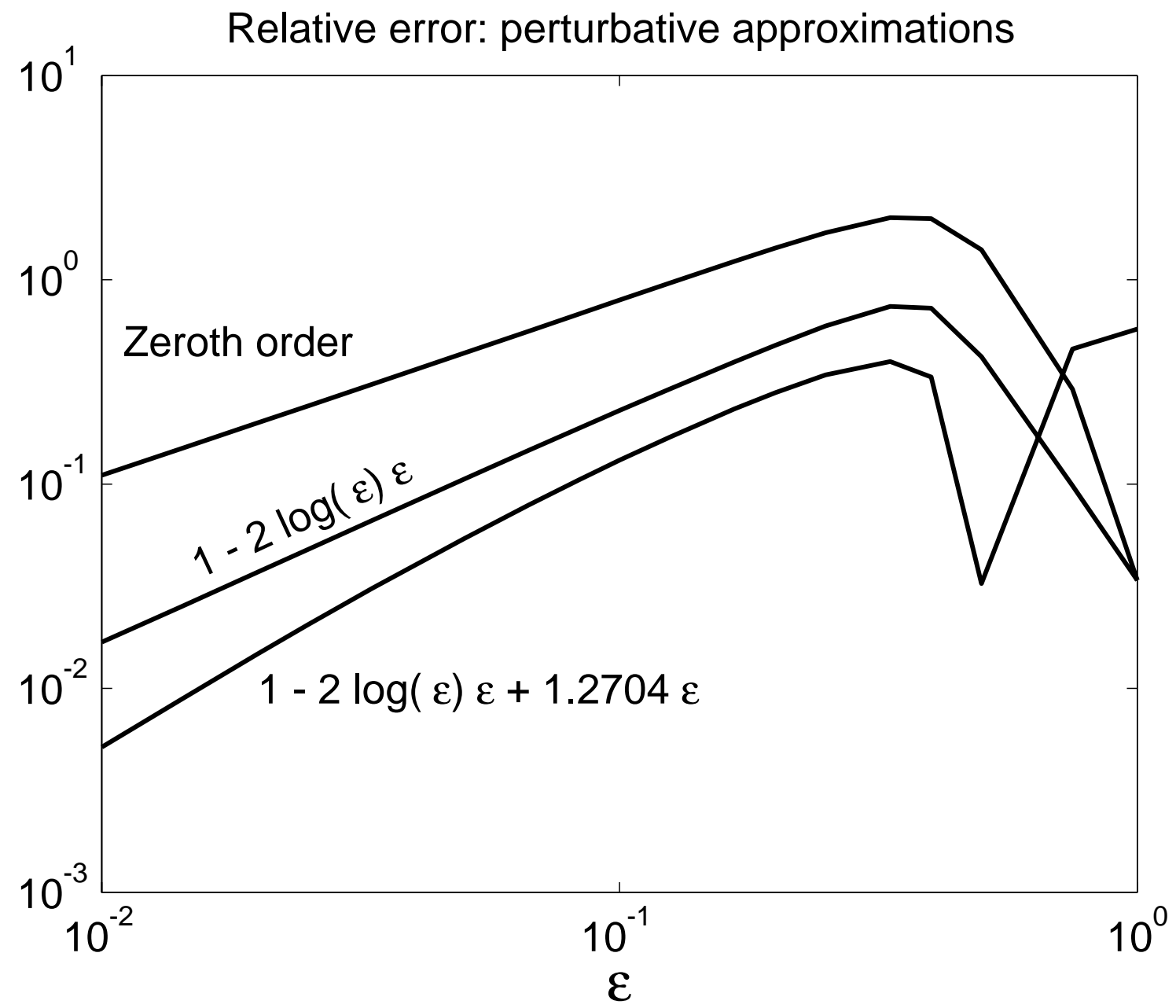

FIG. 3. Relative error $E_{i}, i=0,1,2$.

is much larger than usual for lowest order matched asymptotics [3]. The reason for that is the appearance of $\epsilon \log \epsilon$ at the next order in perturbative approximation. For $\epsilon \sim 1 / 100$

$$
|\epsilon \log \epsilon| \sim 0.05
$$

while

$$
|\epsilon| \sim 0.01
$$

and one should expect relative error to be about 5 times larger than what is expected for a problem in which the answer involves only powers of $\epsilon$. The big errors of the zeroth order approximation motivates extending matched asymptotics to higher order.

The numerical solution is sufficiently accurate even at very small $\epsilon$ that it is possible to determine form of the first correction neglected by our best approximation. The perturbation series for $\Im(\lambda)$ is likely to include terms proportional to

$$
R_{1}(\epsilon)=\epsilon^{2} \log ^{2} \epsilon
$$




$$
\begin{gathered}
R_{2}(\epsilon)=\epsilon^{2} \log \epsilon \\
R_{3}(\epsilon)=\epsilon^{2}
\end{gathered}
$$

Obviously, if a term proportional to $R_{1}(\epsilon)$ is really present in the perturbation series, the leading error term is going to be proportional to (57) as $\epsilon \rightarrow 0$ since it outweighs two other terms in this limit. But for finite $\epsilon$ the relative error might have considerable contributions from $R_{2}(\epsilon)$ and $R_{3}(\epsilon)$ and we might be seeing the sum of three terms. Finally, it might happen that the term proportional to $R_{1}$ in fact does not appear in the perturbation series.

To examine all these possibilities we plot ratios $E_{2} / R_{i}(\epsilon)$ to see which one asymptotes to a horizontal line as $\epsilon \rightarrow 0$. Fig.4 shows that the leading error term - the first perturbative term we do not calculate - is proportional to $\log ^{2}(\epsilon) \epsilon^{2}$. The two steeply rising curves show that the error is blowing up faster than $\epsilon^{2}$ or even $\log (\epsilon) \epsilon^{2}$.

10. Summary and Future Extensions. Even though our eigenproblem has (i) a coefficient which is infinite at the "critical latitude" and (ii) an eigenvalue whose imaginary part is exponentially small in the reciprocal of the perturbation parameter $\epsilon$, we have nevertheless calculated $\Im(\lambda)$ up to and including the $O(\epsilon \log (\epsilon))$ and $O(\epsilon)$ terms. By developing special numerical algorithms, we have confirmed the perturbation theory by numerically computing $\Im(\lambda)$ for $\epsilon$ as small as $1 / 100$.

Our future goal is to apply these methods to the equatorial Kelvin wave. The crucial first step is to generalize the "optical theorem" to the equatorial shallow water equations. It will then be relatively straightforward to apply matched asymptotics to compute the exponentially small part of the eigenvalue.

Acknowledgments. This work was supported by the National Science Foundation through grant OCE9521133. We thank Stan Jacobs for his help.

A. The Gaussian-Stieltjes Integral. The integral

$$
G_{S}(\epsilon) \equiv \lim _{\delta \rightarrow 0} \int_{0}^{\infty} \frac{\exp \left(-t^{2}\right)}{t-[1+i \delta] / \epsilon} d t
$$

cannot be evaluated in closed form. However, because the limit $\delta \rightarrow 0$ of $\delta /\left(\delta^{2}+z^{2}\right)$ is $\pi$ times the Dirac delta-function, the imaginary part of the integral gives

$$
\Im\left(G_{S}\right)(\epsilon)=\pi \exp \left(-\frac{1}{\epsilon^{2}}\right)
$$

without approximation. Thus, this integral is related to the eigenvalue of the Hermitewith-Pole equation in the sense that it has an imaginary part which is a Gaussian function of $1 / \epsilon$.

The real part of the integral can be approximated by the asymptotic series

$$
G_{S}(\epsilon) \sim \sum_{n=1}^{\infty} b_{n} \epsilon^{n}
$$

where, by expanding the denominator of the integrand as a power series in $\epsilon$,

$$
b_{j} \equiv-\int_{0}^{\infty} \exp \left(-t^{2}\right) t^{j-1} d t=-\frac{\Gamma\left(\frac{j}{2}\right)}{2}, \quad j=1,2, \ldots
$$




\section{Relative error of $O(\varepsilon)$ approx./functions of $\varepsilon$}

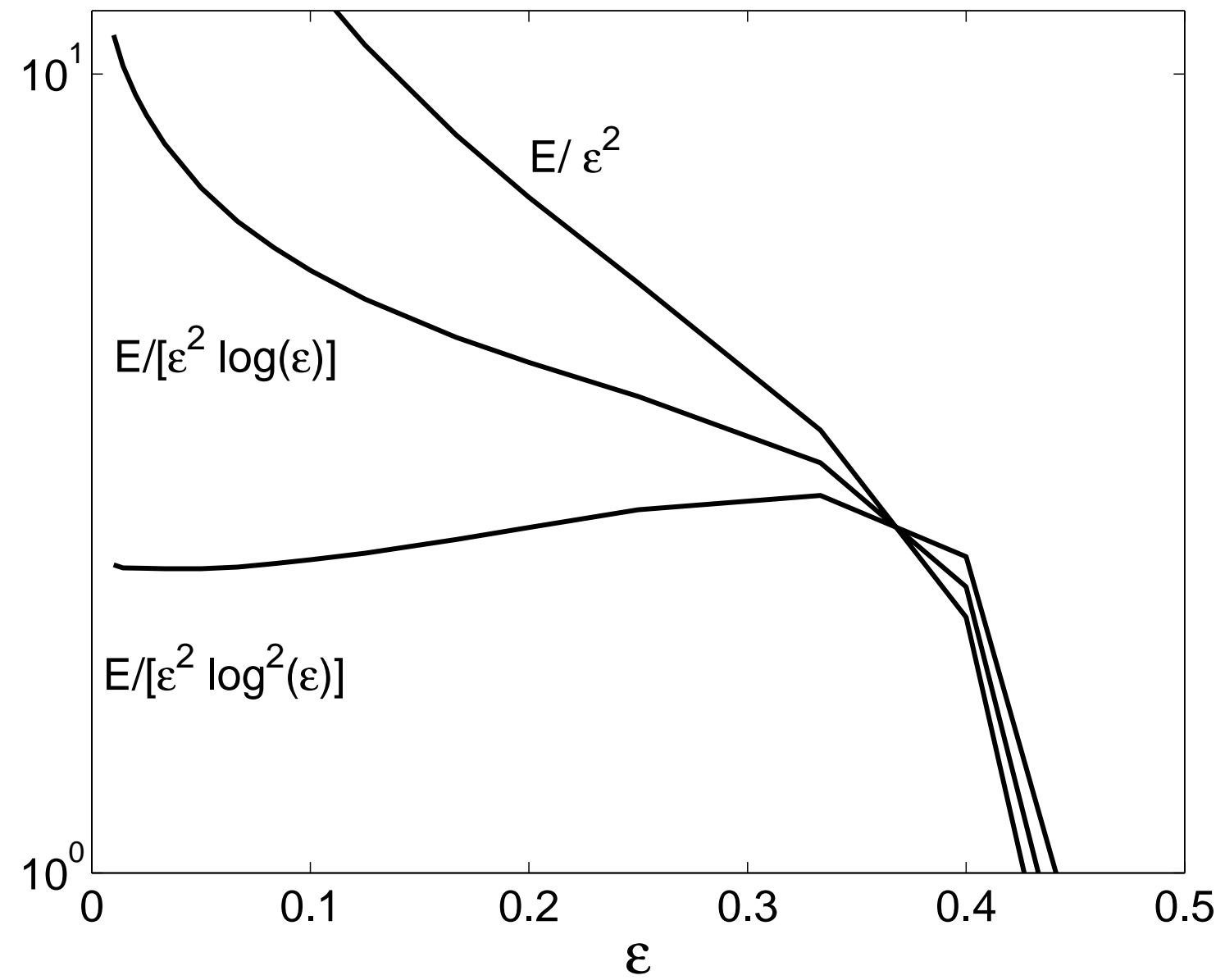

FIG. 4. The relative error in our best approximation is divided by various "gauge" functions. If and only if the guage function correctly matches the leading term in the error, then the ratio of error/gauge function will tend to a constant as $\epsilon \rightarrow 0$.

where $\Gamma$ is the usual factorial function.

Divergent series usually have terms that diverge as $j$ !; the error of an optimallytruncated series is then $O(\exp (-$ constant $/ \epsilon))$. For the Gaussian-Stieltjes integral, $\Im\left(G_{S}\right)$ is proportional to $\exp \left(-1 / \epsilon^{2}\right)$ and the series diverges as the square root of $j !$ (Note that $\log ((j / 2) !) \sim \log (\sqrt{j !})$. This suggests that the Rayleigh-Schrödinger series for the Hermite-with-Pole eigenproblem diverges as the square root of the factorial, too.

Fig. 5 suggests that this conjecture is true. The ratio of the coefficients for the eigenvalue and for the integral does not tend to one as $j \rightarrow \infty$, suggesting that there is perhaps a logarithmic or fractional power of $j$ which is different between the two series. However, the fact that the two sets of coefficients differ by less than a factor of 2 even for $j=26$ where the coefficients are larger than $10^{9}$ suggests that both series depend similarly on the factorial. 

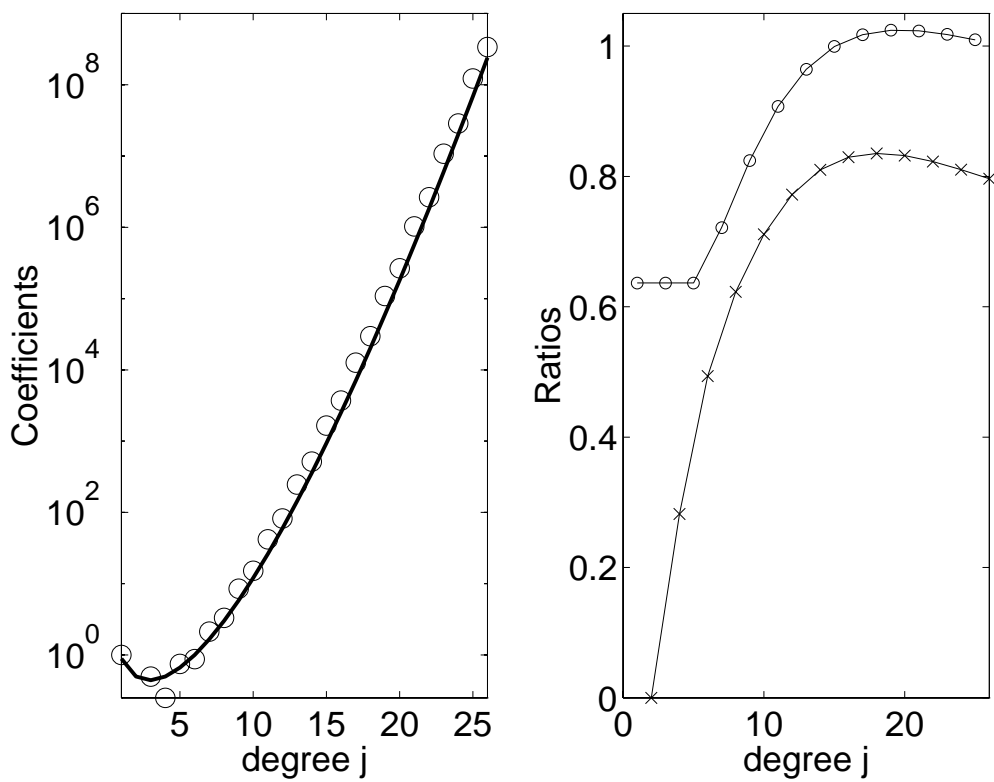

FIG. 5. Left panel: the coefficients of the Gauss-Stieltjes integral (solid line) are compared with those of the eigenvalue series (circles). Right panel: Ratios of the coefficient of the RayleighSchrödinger series for the ground state eigenvalue of the Hermite-singular equation divided by those of the Gauss-Stieltjes integral, $b_{j}$, multiplied by $\pi^{1 / 2}$. Upper curve (circles): odd coefficients $\left\{b_{1}, b_{3}, \ldots\right\}$. Lower curve ( $x$ 's): even coefficients $\left\{b_{2}, b_{4}, \ldots\right\}$.

\section{REFERENCES}

[1] J. A. Adams, Critical layer singularities and complex eigenvalues in some differential equations of mathematical physics, Physics Reports, 142 (1986), pp. 263-356.

[2] D. G. Andrews, J. R. Holton, And C. B. Leovy, Middle Atmosphere Dynamics, Academic Press, New York, 1987.

[3] C. M. Bender and S. A. Orszag, Advanced Mathematical Methods for Scientists and Engineers, McGraw-Hill, New York, 1978. 594 pp.

[4] M. V. Berry, Asymptotics, superasymptotics, hyperasymptotics, in Asymptotics Beyond All Orders, H. Segur, S. Tanveer, and H. Levine, eds., Plenum, Amsterdam, 1991, pp. 1-14.

[5] J. P. Boyd, The effects of latitudinal shear on equatorial waves, Part I: Theory and methods, Journal of the Atmospheric Sciences, 35 (1978), pp. 2236-2258.

[6] - The effects of latitudinal shear on equatorial waves, Part II: Applications to the atmosphere, Journal of the Atmospheric Sciences, 35 (1978), pp. 2259-2267.

[7] — A Sturm-Liouville eigenproblem with an interior pole, Mathematics of Computation, 22 (1981), pp. 1575-1590.

[8] — The optimization of convergence for Chebyshev polynomial methods in an unbounded domain, Journal of Computational Physics, 45 (1982), pp. 43-79.

[9] - Complex coordinate methods for hydrodynamic instabilities and Sturm-Liouville problems with an interior singularity, Journal of Computational Physics, 57 (1985), pp. 454471.

[10] - Spectral methods using rational basis functions on an infinite interval, Journal of Computational Physics, 69 (1987), pp. 112-142.

[11] Chebyshev and Fourier Spectral Methods, Springer-Verlag, New York, 1989. 792 pp.

[12] J. P. Boyd AND Z. D. Christidis, Low wavenumber instability on the equatorial beta-plane, Geophysical Research Letters, 9 (1982), pp. 769-772.

[13] - Instability on the equatorial beta-plane, in Hydrodynamics of the Equatorial Ocean, J. Nihoul, ed., Elsevier, Amsterdam, 1983, pp. 339-351. 
[14] A. W. Gill And G. E. Sneddon, Complex mapped matrix methods in hydrodynamic stability problems, Journal of Computational Physics, 122 (1995), pp. 13-24.

[15] A. W. Gill And G. E. Sneddon, Pseudospectral methods and composite complex maps for near-boundary critical latitudes, Journal of Computational Physics, 129 (1996), pp. 1-7.

[16] R. B. PARIS AND A. D. Wood, Stokes phenomenon demystified, IMA Bulletin, 31 (1995), pp. 21-28. 